\title{
An impedimetric molecularly-imprinted biosensor for Interleukin-1 $\beta$ determination, prepared by in-situ electropolymerization on carbon screen-printed electrodes
}

\author{
Ana R. Cardoso, M.H. de Sá, M. Goreti F. Sales * \\ BioMark-CEB/ISEP, School of Engineering, Polytechnic Institute of Porto, Portugal \\ CEB, Biological Engineering Centre, Minho University, Portugal
}

\section{A R T I C L E I N F O}

\section{Article history:}

Received 1 February 2019

Received in revised form 20 April 2019

Accepted 20 April 2019

Available online 24 April 2019

\section{Keywords:}

Interleukin-1 $\beta$ (IL-1 $\beta$ )

Molecularly imprinted polymer (MIP)

Biosensor

Electropolymerization

Screen-printed carbon electrodes (SPCEs)

Electrochemical impedance spectroscopy (EIS)

\begin{abstract}
A B S T R A C T
This work reports the first electrochemical molecularly imprinted polymer (MIP) sensor for Interleukin-1beta (IL-1ß) detection, based on modified commercial screen-printed carbon electrode (SPCE) was successfully demonstrated. For this purpose, the carbon support was modified with a PEDOT/4-aminothiophenol layer prior to the MIP film to enhance sensitivity and signal stability. The MIP layer was constructed on top of this by electropolymerization of Eriochrome black T (EBT) in the presence of IL-1 $\beta$. The several steps of the biosensor assembly was followed by Raman spectroscopy and electroanalytical techniques.

Using electrochemical impedance spectroscopy (EIS), a linear response in the range of $60 \mathrm{pM}$ to $600 \mathrm{nM}$, with a LOD of $1.5 \mathrm{pM}$ with $(\mathrm{S} / \mathrm{N}=3)$ was obtained in neutral PBS. Selectivity tests of the MIP biosensor made in spiked synthetic serum samples as well as against other structurally related (Myoglobin, of similar shape and size) or competing compounds (Immunoglobulin G, also present in the human serum) confirmed the good selectivity of the biosensor.

Overall, the biosensor described herein has the potential to provide a simple and quick way for on-site screening of IL- $1 \beta$, with low sample/reagent consumption and enabling direct serum analysis, which constitutes a valuable alternative to other conventional methods.
\end{abstract}

(c) 2019 Elsevier B.V. All rights reserved.

\section{Introduction}

The role of inflammation in cancer is controversial as both tumourpromoting and tumour-suppressive aspects of inflammation have been reported. Interleukin-1 $\beta$ (IL-1 $\beta$ ), a pro-inflammatory cytokine is implicated in cancer cell proliferation [1], emerging now as a key mediator of carcinogenesis. Its involvement in the molecular pathways of cancer-related inflammation are being unravelled, especially in pancreatic [2], breast [3] and prostate cancers [4]. Monitoring this biomarker in point-of care may therefore contribute to follow-up the progress of cancer diseases.

The standard technique for the detection of IL-1 $\beta$ is enzyme-linked immunosorbent assay (ELISA), which is capable o detecting $0.2 \mathrm{pg} / \mathrm{mL}$ of this biomarker [5]. Although this technique is highly sensitive and accurate, it is still time-consuming, requires specialized personnel and relatively large sample volumes. This means that alternative procedures are urgently required.

In the context of biochemical sensing, electrochemical biosensors are today particularly relevant, especially when using miniaturized 3-

\footnotetext{
* Corresponding author.

E-mail address: mgf@isep.ipp.pt (M.G.F. Sales).
}

electrode systems, as screen-printed electrodes (SPEs) [6]. SPEs combine in a small spot the required electrodes, allowing point-of-care analysis when coupled to suitable electronic device that collects the electrical signal (similar to the glucose meter). In terms of composition, SPEs may have working electrodes of different materials, including gold, carbon or platinum [7]. Among these, carbon supports (SPCEs) are very popular, being highly effective and of low cost [8]. As far as we know, there is a single electrochemical biosensor work published for IL-1 $\beta$, which is an immunosensor assembled on double walled carbon nanotubes showing limits of detection of $0.38 \mathrm{pg} / \mathrm{mL}$ [9]. Yet, this biosensor uses commercial antibodies as biorecognition element, which increases the cost of the overall process, and decreases the stability of the device and its lifetime.

As an advantageous alternative to antibodies, biomimetic materials may be tailored by molecular imprinting technology [10]. In this, a molecularly-imprinted polymer (MIP) requires placing the molecule to be imprinted among specific monomeric compounds and starting the polymerization by a suitable stimulus. The removal of the imprinted molecules yields the formation of cavities that act as antibodies, for displaying a complementary shape to the target molecule. The use of electrical stimulus to initiate the polymerization (electropolymerization) is increasing in the literature, because it allows 
the production a homogeneous and thin MIP film directly on the electrode surface, requiring very little amount of reagents and within few minutes/s [11]. Moreover, this procedure is highly reproducible and allows an easy control of the polymer thickness [12].

There are several monomers that may be employed to electrically generate MIP materials, which are typically aromatic compounds with different functionalities $[13,14,15]$. In this regard, our group has pioneered the use of Eriochrome Black T (EBT) as functional monomer, which yielded very stable and controllable polymeric surfaces $[16,17]$. EBT has several functional groups, e.g., sulfonate, hydroxyl, nitrogen dioxide, and diazo, enabling different interactions with a given target analyte, and it is classified as an anionic acidic dye with highly extended $\pi$ system. Moreover, as response to the electrical stimulus the electrogenerated poly-Eriochrome Black T (PEBT) on the surface of the electrode has a high concentration of the negatively-charged functional group $\left(-\mathrm{SO}_{3}^{-}\right)$and also a high number of electron-rich oxygen atoms on its surface [18].

Thus, this work proposes for the first time the production of a MIP material to target the protein IL-1 $\beta$, by employing electropolymerization of EBT monomers on SPCEs. Herein, the carbon support was first modified with 3,4-ethylenedioxythiophene (EDOT) [16] and after modified with 4-aminothiophenol (4-AMP) which acted as linker between the PEDOT film and the subsequent MIP layer [17]. The biosensor was evaluated by electrochemical impedance spectroscopy (EIS), optimized and tested on buffer and serum systems.

\section{Experimental section}

\subsection{Reagents}

All chemicals were of analytical grade and ultrapure Milli-Q water laboratory grade (conductivity $<0.1 \mu \mathrm{S} / \mathrm{cm}$ ) was used. The chemical reagents used included potassium hexacyanoferrate (III) $\left(\mathrm{K}_{3}\left[\mathrm{Fe}(\mathrm{CN})_{6}\right]\right)$ and potassium hexacyanoferrate (II) trihydrate $\left(\mathrm{K}_{4}\left[\mathrm{Fe}(\mathrm{CN})_{6}\right] .3 \mathrm{H}_{2} \mathrm{O}\right)$, obtained from Riedel-de Häen; Phosphate buffered saline (PBS) tablets purchased from Sigma-Aldrich; 3,4-Ethylenedioxythiophene, 97\% (EDOT), from Alfa Aesar; 4-Aminothiophenol, 96\% (4-AMP), from Acros Organics; EriochromeBlack T (EBT), from Biochem; Interleukin$1 \beta$ (IL-1ß) (AA 117-269) with purity $>95 \%$ in PBS pH 7.4 (concentration $1 \mathrm{mg} / \mathrm{mL}$ ) from Antibodies-online, Germany. Myoglobin (Myo) and Immunoglobulin G ( $\operatorname{IgG}$ ) were purchased from Sigma-Aldrich; Glucose (Glu) from Fisher BioReagents and Urea from Fagron; sodium chloride $(\mathrm{NaCl})$ from Panreac; potassium chloride $(\mathrm{KCl})$ and calcium chloride $\left(\mathrm{CaCl}_{2}\right)$ from Merck; magnesium chloride hexa-hydrate $\left(\mathrm{MgCl}_{2} \cdot 6 \mathrm{H}_{2} \mathrm{O}\right)$ from Riedel-de Häen was also used.

\subsection{Solutions}

All solutions (except 4-AMP) were prepared in ultrapure water. The PBS (0.1 M, pH 7.49) was prepared by dissolution of the commercial tablets. A $1.0 \times 10^{-3} \mathrm{M} 4$-AMP solution was prepared in a 3:7 (v:v) EtOH: $\mathrm{H}_{2} \mathrm{O}$ solution. A $10 \times 10^{-3} \mathrm{M}$ EDOT solution was prepared in $0.1 \mathrm{M}$ PBS, as well as the solution of $1.0 \times 10^{-3} \mathrm{M}$ EBT and $3 \times 10^{-6}$ M IL-1 $\beta$ used for imprinting. Stock standard solutions of $100 \mu \mathrm{g} / \mathrm{mL}$ $\left(6.0 \times 10^{-6} \mathrm{M}\right)$ of IL-1 $\beta$ were prepared in PBS and stored at $-20{ }^{\circ} \mathrm{C}$. Less concentrated $\left(6.0 \times 10^{-11}\right.$ to $\left.6.0 \times 10^{-7} \mathrm{M}\right)$ IL- $1 \beta$ standard solutions were needed to calibrate the biosensor and were prepared by accurate dilution of the stock solution in PBS. The impedance response was recorded for each concentration by covering in a drop-like fashion the biosensors with an electron mediator solution of $5.0 \times 10^{-3} \mathrm{M}$ of potassium hexacyanoferrate III, $\left(\mathrm{K}_{3}\left[\mathrm{Fe}(\mathrm{CN})_{6}\right]\right)$, and $5.0 \times 10^{-3} \mathrm{M}$ of potassium hexacyanoferrate II $\left(\mathrm{K}_{4}\left[\mathrm{Fe}(\mathrm{CN})_{6}\right] .3 \mathrm{H}_{2} \mathrm{O}\right)$, in $0.1 \mathrm{M}$ PBS. The initial "cleaning" procedure of the working electrode area required a $0.5 \mathrm{M}$ $\mathrm{H}_{2} \mathrm{SO}_{4}$ aqueous solution.

Interfering solutions of Myoglobin (Myo) and Immunoglobulin G $(\operatorname{IgG})$ were prepared in PBS, with A concentration $6.0 \times 10^{-9} \mathrm{M}$, as
IL-1 $\beta$. Synthetic serum was also prepared according to [19] and consisted of $\mathrm{NaCl}\left(145 \times 10^{-3} \mathrm{M}\right), \mathrm{KCl}\left(4.5 \times 10^{-3} \mathrm{M}\right), \mathrm{CaCl}_{2}(32.5$ $\left.\times 10^{-3} \mathrm{M}\right), \mathrm{MgCl}_{2}\left(0.8 \times 10^{-3} \mathrm{M}\right)$, Urea $\left(2.5 \times 10^{-3} \mathrm{M}\right)$, and Glucose $\left(4.7 \times 10^{-3} \mathrm{M}\right)$ in aqueous solution.

\subsection{Electrochemical apparatus}

Electrochemical characterization was performed at room temperature using a PalmSens portable $(10.0 \times 6.0 \times 3.4 \mathrm{~cm}$ dimensions $)$ potentiostat/galvanostat, EmStat4 Blue, integrating an EIS analyzer module. This device was controlled by the PSTrace 5.3 software (PalmSens, Netherlands). The SPCEs, DRP-110, were purchased from DropSens, Inc. (Asturias, Spain), and consisted of a carbon counter/auxiliary electrode, a silver pseudo-reference electrode and a carbon working electrode of $4 \mathrm{~mm}$ diameter in a strip of a ceramic substrate (3.4 $\times 1.0 \times 0.05 \mathrm{~cm}$ dimensions). All potential values were referred to the screen-printed silver pseudo-reference electrode. SPCEs were connected to the potentiostat via a switch box from BioTID eletrónica, Portugal $(8.0 \times 4.0 \times 3.5 \mathrm{~cm}$ dimensions $)$.

\subsection{Electropolymerized MIP biosensor assembly on SPCE}

The assembly of the MIP on the SPCE was obtained by electropolymerization by "bulk" imprinting, in a PBS solution containing IL-1 $\beta$ and EBT. This was achieved by using chrono-amperometry (CA) at $+0.90 \mathrm{~V}$ for $200 \mathrm{~s}$, after testing different time lengths $(100,150$ and $200 \mathrm{~s}$ ), in order to control the polymeric layer thickness by the amount of charge passed. Prior to this procedure, some modifications of the bare SPCE had to be performed, as illustrated in Scheme 1. First the SPCE was rinsed with ultrapure water and then cleaned in $0.5 \mathrm{M} \mathrm{H}_{2} \mathrm{SO}_{4}$ by cyclic voltammetry $(\mathrm{CV})$, between $-0.2 \mathrm{~V}$ and $+1.5 \mathrm{~V}$, scanning at $50 \mathrm{mV} / \mathrm{s}$, during 5 cycles. After this, the carbon surface was washed with ultrapure water and the next step consisted in the electrochemical polymerization of EDOT by CA at $+0.9 \mathrm{~V}$ for 10 s, followed by incubation in 4AMP for $1 \mathrm{~h}$. Only then the MIP layer was produced by "bulk" electropolymerization of EBT in the presence of IL-1 $\beta$. The last step consisted in removing electrochemically the template from the surface of the modified SPCE by scanning CV cycles in $0.1 \mathrm{M}$ PBS, from $-0.30 \mathrm{~V}$ to $+0.80 \mathrm{~V}$, with a scan rate of $0.10 \mathrm{~V} / \mathrm{s}$ for 25 cycles. For comparison purposes, control SPCEs were produced by synthesising non-imprinted polymer (NIP) films, following the same steps described before, but in the absence of the protein template (IL-1 $\beta$ ).

\subsection{Electrochemical characterization of the biosensor}

All electrochemical assays were made in duplicate. The changes in the electrical properties of the sensing surface were monitored by the response of the redox probe ferri/ferrocyanide, $\left[\mathrm{Fe}(\mathrm{CN})_{6}\right]^{3-/ 4-}$, in order to deliberately introduce an electron transfer process at the SPCE surface and in this way characterize the surface. Voltammetric techniques like CV and square wave voltammetry (SWV), and also EIS were performed to this end. In CV and SWV assays, potentials were scanned from -0.3 to $+0.7 \mathrm{~V}$ at $0.050 \mathrm{~V} / \mathrm{s}$, and -0.2 to $+0.5 \mathrm{~V}$ at $0.020 \mathrm{~V} / \mathrm{s}$, respectively. This was a quick and easy approach to identify how the electrode surface modification affected the probe redox processes and their behavior [20,21]. For further characterization, EIS assays were performed at the open circuit potential (OCP), by using a sinusoidal potential perturbation with an amplitude of $0.01 \mathrm{~V}$ and 50 data points acquisition, logarithmically distributed over $0.01-100,000 \mathrm{~Hz}$ frequency range. The EIS data was analyzed by Nyquist plots (frequency response of the electrode/electrolyte system and area plot of the imaginary component ( $\left.Z^{\prime \prime}\right)$ of the impedance, against the real component $\left(Z^{\prime}\right)$. All EIS data was fitted to a Randle's equivalent circuit, using the PSTrace 5.3 software (Palm Sens, Netherlands), aiming to retrieve the charge-transfer resistance $\left(R_{c t}\right)$ of the surface. The $R_{c t}$ value can be used to detect surface modifications since it reflects the energy 


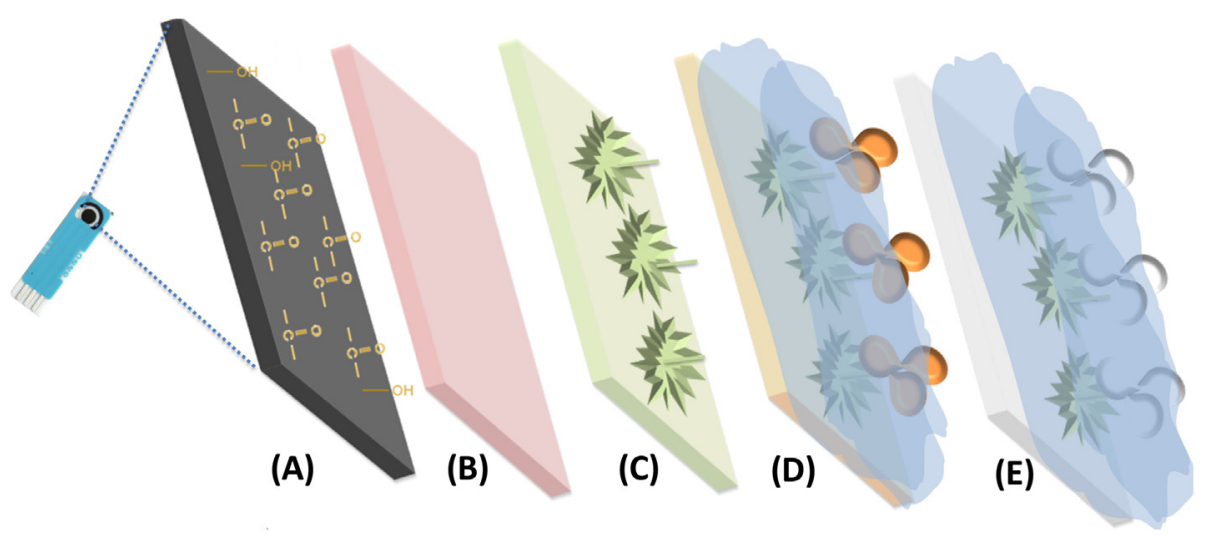

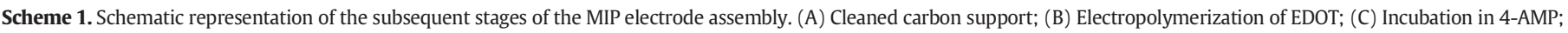
(D) Electropolymerization of EBT in the presence of IL-1B; (E) Template removal.

barrier of the redox species reaching the electrode, due to electrostatic repulsion or steric hinderance [22]. Visually, an increase in $\mathrm{R}_{\mathrm{ct}}$ can be seen in the Nyquist plot as an increase in the diameter of the semicircle portion of the curve.

Calibration curves were made for testing the MIP and NIP films template rebinding and used EIS after incubation of each standard solution

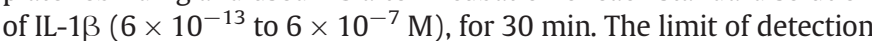
(LOD) was calculated as the concentration extracted from the linear response corresponding to $(x+3 \sigma)$, where $x$ was the average value of the blank (buffer) signals and $\sigma$ the corresponding standard deviation [17].

Selectivity studies also used EIS. These studies involved the analysis of the response of the biosensor after 30 min incubation with IL-1 $\beta$ spiked synthetic serum, in the linear range of concentrations applied in the calibration curves, in order to evaluate matrix effects. Moreover, the competing proteins effect by using binary assays between IL-1 $\beta$ $\left(6.0 \times 10^{-9} \mathrm{M}\right)$ and a different protein in the same concentration (interfering proteins selected were Myo and $\operatorname{IgG}$ ) was also evaluated.

\subsection{Raman characterization of the biosensor}

Raman spectroscopy was performed in order to further characterize the morphology of the biosensor assembled on the SPCE. This was done by direct analysis of the working electrode region of the prepolymerized, MIP and NIP modified SPCEs in a Thermo Scientific Raman DXR spectroscope, equipped with a $532 \mathrm{~nm}$ laser. The average signal-to-noise ratio (peak height/RMS noise) was allowed for $900 \mathrm{~s}$ measurement time, using a $0.5 \mathrm{~mW}$ laser power and a $50 \mu \mathrm{m}$ slit aperture. Raman spectra were collected in the range $3500-100 \mathrm{~cm}^{-1}$. To ensure that the data was representative of the overall film quality, several spectra were collected on different sample locations at the highest magnification (objective 50×). A pristine unmodified SPCE was used as control.

\subsection{AFM characterization of the surface of the biosensor}

The 3D topography images and roughness measurements of the MIP and NIP modified SPCE surfaces were evaluated by Atomic Force Microscopy (AFM). AFM 3D images were obtained with a PicoPlus 5500 scanning probe microscope (Keysight Technologies, USA) using the PicoView 1.20 software (Keysight Technologies, USA). Each sample was imaged with a $10 \times 10 \mu \mathrm{m}^{2}$ piezoelectric scanner on three randomly chosen locations of each sample. All measurements were performed in TappingTM mode at RT in liquid $\left(\mathrm{H}_{2} \mathrm{O}\right)$, using V-shaped cantilever silicon tips (HYDRA-ALL-G, AppNano, USA) with a spring constant of $0.389 \mathrm{~N} / \mathrm{m}$. Scan speed was set at 0.5 line/s. The roughness measurements results were obtained using the WSXM 5.0 software [23], where the values of RMS (root mean square) were determined.

\section{8. pI characterization of IL-1ß by LC-MS/MS}

The commercial protein IL-1 $\beta$ isoelectric point ( $\mathrm{pI}$ ) was inferred from the high-resolution accurate mass spectrometry, LC-MS/MS analysis of the sample. The protein sample was digested with trypsin, and the IL-1 $\beta$ sample tryptic peptides were analyzed by nanoLC-MS/MS using an Ultimate 3000 liquid chromatography system coupled to a QExactive Hybrid Quadrupole-Orbitrap mass spectrometer (Thermo Scientific, Bremen, Germany). The raw data was processed using Proteome Discoverer 2.2.0.388 software (Thermo Scientific) and searched against the UniProt database for the taxonomic selection Homo sapiens (September 2017 release) and SwissProt database, All entries (October 2017 release). The Sequest HT search engine was used to identify tryptic peptides. Peptide confidence was set to high.

\section{Results and discussion}

In general, the assembly of a MIP for IL-1 $\beta$ on SPCEs followed the schematic representation in Scheme 1 . For this purpose, a pretreatment was first established, as the blank carbon support was not stable enough and did not have the necessary electrical and chemical features. The MIP and control films were assembled next and followed-up by different analytical techniques.

\subsection{SPCE pre-treatment}

The first pre-treatment approach included a cleaning stage to ensure the electrochemical oxidation of all materials/impurities standing at the working electrode carbon surface. This was made by consecutive CV cycling in a solution of $0.5 \mathrm{M} \mathrm{H}_{2} \mathrm{SO}_{4}$ [16]. After this, the signal of the redox probe mediator corresponded to a slight current increase in SWV, when compared to bare SPCEs (as acquired, unmodified). This current increase was coupled to a small decrease in $\mathrm{R}_{\mathrm{ct}}$ (EIS) and a lower potential difference between oxidation and reduction peaks of the probe (CV), as shown in Fig. S1. Therefore, this cleaning procedure enhanced the electron transfer capability of each bare SPCE and contributed for a higher reproducibility among the different commercial units of SPCEs tested, in agreement with previous studies $[16,17]$.

The second pre-treatment approach aimed at improving the electrical response of the biosensor, and consisted in the electropolymerization of EDOT, to generate PEDOT, which is amongst the most stable conducting polymers currently available [24]. This was achieved by applying a fixed potential to an EDOT solution, as previously described [25]. After this, the working electrode surface of the SPCE should be covered by a thin-film of the highly conductive polymer PEDOT. The resulting surface was evaluated by the electrochemical techniques CV, EIS and SWV, shown in Fig. S1, which confirmed the 
presence of a highly conductive film on top of carbon. The resulting CV and SWV data revealed a significant increase in the current value of the redox probe, when compared to the oxidized SPCEs. Consistently, the Nyquist plot had no longer a semicircle, thereby confirming the enhanced conducting electrical features of the SCPE/PEDOT (Fig. S1).

A third pre-treatment approach established before the MIP imprinting consisted in adding a layer to which the monomeric species EBT of the MIP film could attach, thereby generating a steady biosensor. In this, the PEDOT layer was modified by incubation in 4-AMP. Overall, the 4-AMP acted as a linker between the PEDOT layer and the subsequent PEBT film. It contained an -SH bond that could favour its binding to PEDOT, and an aromatic amine that could also be involved in the polymerization of EBT. It is important to mention that if a disulphide bond would not be formed on the PEDOT layer spontaneously (which is not likely to happen because this reaction was followed previously by us on a glass support and the colour of the PEDOT layer changed after the addition of 4-AMP), the aromatic ring with an amine function in 4AMP could always react with both carbon layer and EBT upon the electrochemical conditions applied next. This step was not electrochemically monitored, in order to avoid the electropolymerization of 4-AMP, which would decrease the availability of chemical functionalities and potential binding sites for the EBT [17]. Finally, EBT is an electroactive species that contains diazo $(-\mathrm{N}=\mathrm{N}-)$ and hydroxyl groups bound to aromatic rings, which are likely to be involved in the electropolymerization of PEBT.

\subsection{Assembly of MIP and NIP modified SPCE}

In order to identify the best conditions to carry out the electropolymerization of EBT, the SPCE/PEDOT/4-AMP support underwent $\mathrm{CV}$ experiments within a large potential range (from -1.0 to $+1.0 \mathrm{~V}$, at $50 \mathrm{mV} / \mathrm{s}$ ), using single component solutions of 1.0 $\times 10^{-3} \mathrm{M}$ EBT or $6.0 \times 10^{-6} \mathrm{M}$ IL-1 $\beta$, prepared in PBS. This study would indicate the best potential to carry out the electropolymerization of EBT and ensure that the target protein would not be co-polymerizing with the PEBT formed in situ. The results obtained are depicted in Fig. 1 and show that the electrochemical features of these individual components on the SPEC/PEDOT/4-AMP were rather similar, which was unexpected. However, comparing with the blank (SPCE/PEDOT/4-AMP) and the already reported [16] data regarding the electro-oxidation behaviour of EBT in PBS, one realises that the relevant peaks were being

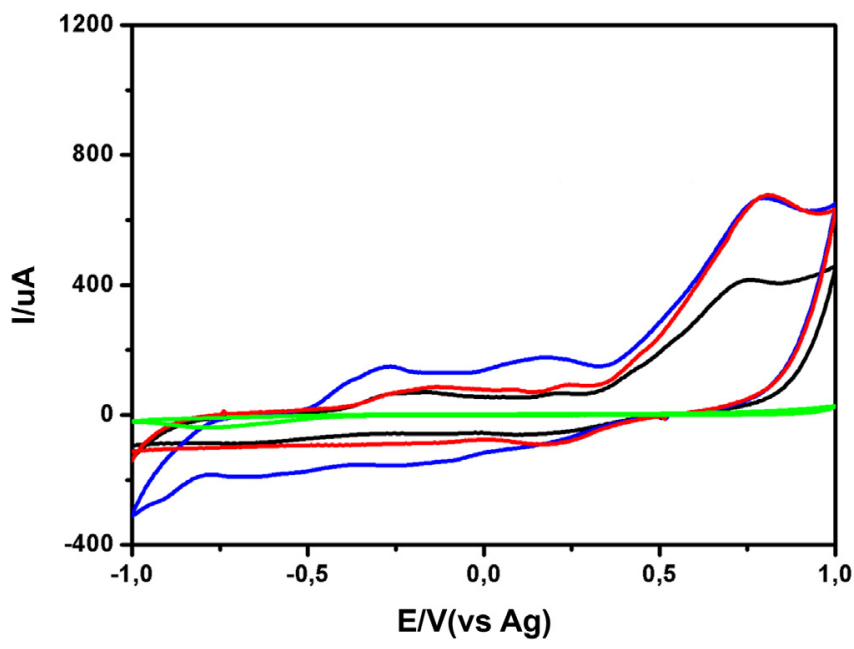

Fig. 1. Cyclic voltammograms in PBS at $50 \mathrm{mV} / \mathrm{s}$ of the (PEDOT/4-AMP) modified SPCE (black line) in the presence of $1 \times 10^{-3} \mathrm{M}$ EBT (blue line) or $6 \times 10^{-6} \mathrm{M} \mathrm{IL}-1 \beta$ (red line), including also the response of $6 \times 10^{-6} \mathrm{M}$ IL- $1 \beta$ on the surface of the polymerized PEDOT in the absence of 4-AMP modified SPCE (green line). (For interpretation of the references to colour in this figure legend, the reader is referred to the web version of this article.) generated by the 4-AMP layer. To check this hypothesis, an additional CV assay of the IL-1 $\beta$ solution was performed on a surface of SPCE/ PEDOT (Fig. 1), and the observed peaks were suppressed. This confirmed the stability of the protein over the scanned potential window and indicated that the peaks of the scan of the IL- $1 \beta$ solution on the 4AMP modified surface reflected the presence of 4-AMP itself. The potential selected for the electropolymerization of EBT was $+0.9 \mathrm{~V}$, ensuring a good rate of polymer formation and reproducibility among different biosensing units.

MIP films were obtained by bulk polymerization of a PBS solution containing $3.0 \times 10^{-6} \mathrm{M}$ IL-1 $\beta$ and $1.0 \times 10^{-3} \mathrm{M}$ EBT, by applying $+0.90 \mathrm{~V}$ at the SPCE/PEDOT/4-AMP. NIP films were prepared similarly, by using instead a solution of only $1.0 \times 10^{-3} \mathrm{M}$ EBT. The possibility of having polymeric films of different thicknesses was tested by applying the selected potential for different timings: 100,150 and 200 s. The overall CV, EIS and SWV data of the modified SPCE with the MIP layer with different polymerization times are shown in Fig. S2. In these, the current intensity of the redox peaks of the probe at the SPCEs in CV and SWV decreased after growing the polymeric layer for longer times. As expected, this was coupled to a higher peak-to-peak potential separation in CV, due to a more blocked electrode surface [20]. The results in EIS were also consistent with these observations, with $\mathrm{R}_{\mathrm{ct}}$ increasing more evidently in the MIP formed after 200 s. This could be attributed to the thickness of the film and the electrostatic repulsion force between the negatively charged $\left[\mathrm{Fe}(\mathrm{CN})_{6}\right]^{3-/ 4-}$ and the EBT film, which in the presence of the template (IL-1 $1 \beta$ ) are less dominant due to the protein heterogeneous charge distribution [26]. This is a dual feature since the protein presence may contribute to establish attractive electrostatic interactions with the probe, besides blocking the electrode surface due to its size. Further confirmation of these observations were gathered from the NIP film acting as control.

It is important to highlight that there are many ways to remove the protein from an imprinted film, being the most employed ones chemical- or enzymatic-based procedures. Herein, an electrochemical approach in PBS was implemented for being a simple and effective approach. The superficial protein molecules that are absorbed on the surface were likely to exit the polymeric network successfully and more easily, because this adsorption could be disturbed by ionic buffered species incubated on the electrode surface. This disturbance could be further intensified when the electrode was being polarized alternatively and consecutively with different charges.

Overall, there were clear differences between the MIP and NIP polymeric materials, as shown in Fig. 2. In general, the MIP film evidenced smaller current intensities and particularly higher peak separation in $\mathrm{CV}$, revealing a more blocked electrode surface than the NIP. The EIS confirmed these observations and also revealed to be a better tool to infer the materials modifications. EIS signals are governed by the impedance of the surface and solution interface, which is generally dominated by capacitive contributions [20]. For this reason, it was possible to detect the $\mathrm{R}_{\mathrm{ct}}$ increasing significantly after template removal of the MIP while it remains almost unchanged in the NIP sample. This difference can only be attributed to the important role of the template in the MIP polymerization process. As IL-1 $\beta$ was intercalated within the growing polymer, the polymeric matrix had different electrical properties, with asymmetric charge distribution, and leading to lower electrostatic repulsion force between the negatively charged redox probe and the EBT film. In contrast, IL-1 $\beta$ has a large size $(17 \mathrm{kDa})$ and interfered with the polymer growth and the probe interaction with the electrode surface, therefore justifying that the MIP showed higher $\mathrm{R}_{\mathrm{ct}}$ than the NIP. Afterwards, the template removal led to a further resistance increase, due to the higher electrostatic repulsion force between the negatively charged $\left[\mathrm{Fe}(\mathrm{CN})_{6}\right]^{3-/ 4-}$ and the negatively charged sites at EBT film. These findings confirmed therefore the incorporation and poor electroactive character of the template in the MIP assembly. Overall, all the electrochemical analysis of the biosensor assembly were consistent with a successful polymer formation and efficient template 

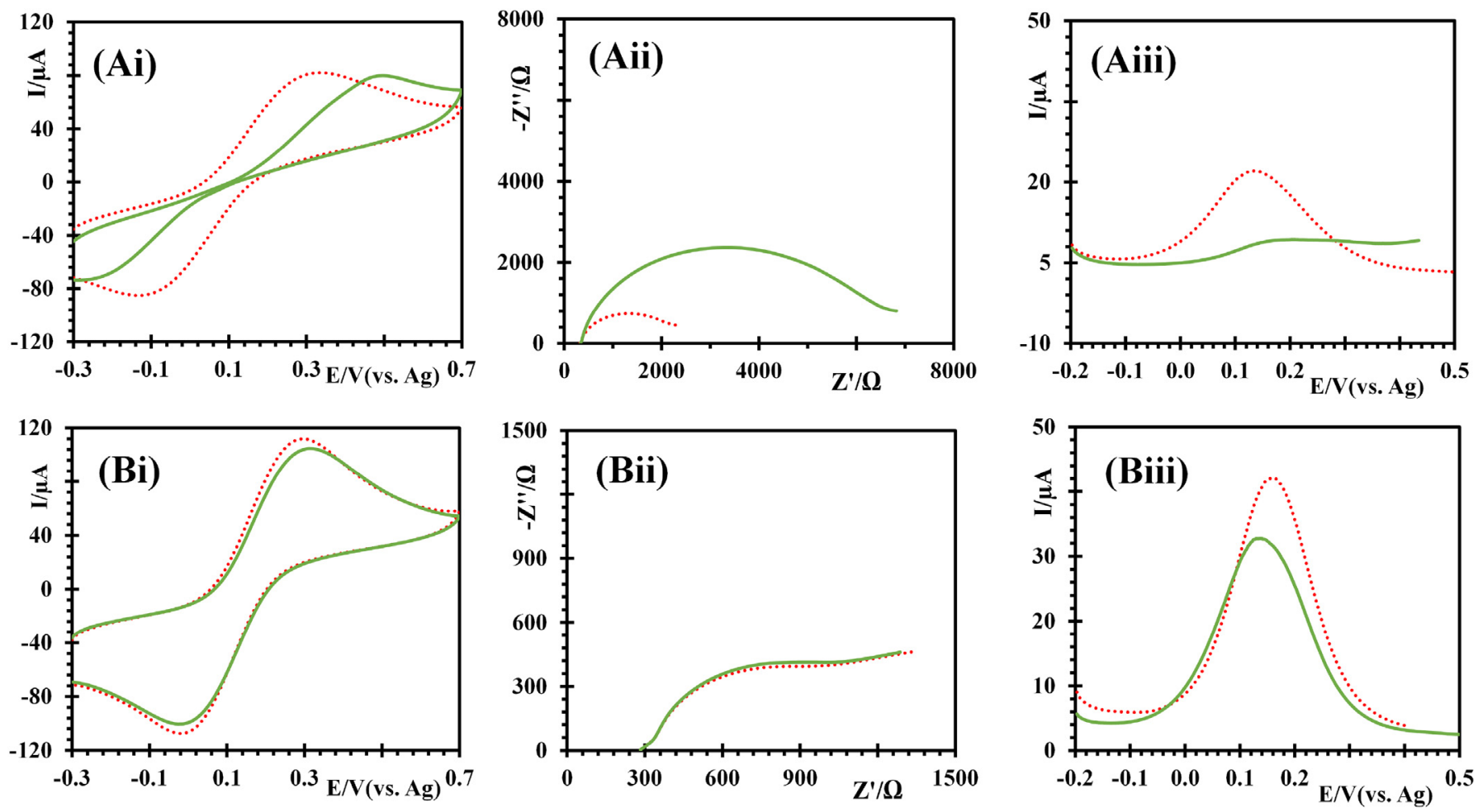

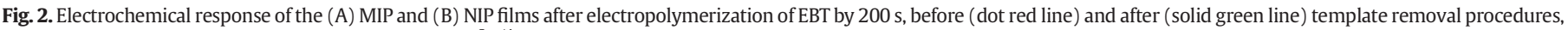

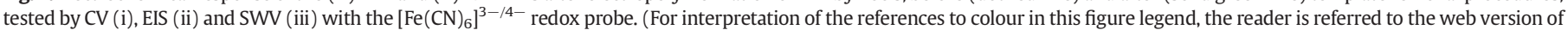
this article.)

removal, being these features more evident for the longer polymerization time tested (200 s).

\subsection{Raman characterization of the biosensor}

It has been shown before that the Raman spectroscopy with a $532 \mathrm{~nm}$ laser can give valuable chemical information about carbon materials and their chemical modification, particularly in the MIP modification of SPCE $[16,17]$. For carbon-based materials, two peaks at approximately $1360 \mathrm{~cm}^{-1}$ (D peak) and $1580 \mathrm{~cm}^{-1}$ (G peak) are typically found, along with a third peak ( $G^{\prime}$ or 2 D peak) located around $2700 \mathrm{~cm}^{-1}$. Both $\mathrm{G}^{\prime}$ and $\mathrm{G}$ peaks are typically found in graphite spectra, and the $\mathrm{D}$ band indicates the defects or disorder in the carbon structure [27]. So, a quantification of the disorder in the materials can be obtained by calculating the ratio between the $\mathrm{D}$ (related to $\mathrm{sp}^{2}$ carbon bonding) and $\mathrm{G}$ (related to $\mathrm{sp}^{3}$ carbon bonding) peaks, and in this way $\left(\mathrm{I}_{\mathrm{D}} / \mathrm{I}_{\mathrm{G}}\right)$ can be used to check the occurrence of a given chemical modification. Furthermore, the EDOT electropolymerization on top of oxidized SPCE, has been associated with the occurrence of two additional peaks that for simplicity were called 1 and 2 and assigned to the symmetric and asymmetric $C_{\alpha}=C_{\beta}$ stretching in the five-member PEDOT ring, around $1434 \mathrm{~cm}^{-1}$ (stronger signal) and $1505 \mathrm{~cm}^{-1}$, respectively [16].

Therefore, MIP and MIP after template removal, as well as NIP modified SPCEs were analyzed by Raman spectroscopy and their spectra compared with the ones obtained for pre-conditioned SPCE (PEDOT/4AMP modified) and the pristine/bare SPCE (Fig. S3). The absolute values of Raman shift, peak intensity, for the major peaks and also the $\mathrm{I}_{\mathrm{D}} / \mathrm{I}_{\mathrm{G}}$ are shown in Table 1.

Overall, results showed that the intensity of the $\mathrm{D}$ peak relative to that of the $G$ peak increased with the amount of disorder introduced within the carbon material after a given chemical modification. One can see from Table 1 that with the exception of the MIP after removal of the template (MIP rem), all the other layers presented higher order $\left(\mathrm{I}_{\mathrm{D}} / \mathrm{I}_{\mathrm{G}}<1\right)$ upon modification, since the $\mathrm{D}$ peak is less intense than $\mathrm{G}$. In addition, the differences in intensity between the Raman spectra of
PEDOT (peaks 1 and 2) clearly defined in the SPCE/PEDOT/4-AMP (Blank) and Raman spectra of the other materials containing PEBT, were a good indicator that the surface was covered by the polymeric material. This confirmed the successful molecularly-imprinted modification of the PEDOT layer. Moreover, the spectra of the MIP film after template removal (MIP rem) showed increased evidence of the PEDOT film, revealing that the protein was no longer on the polymeric layer. Moreover, it was very difficult to recognize the PEDOT signature in the NIP, which is consistent with the fact that the NIP modified SPCE should have a ticker film of polymeric material on top of the PEDOT/4-AMP layer, providing also a strong evidence of the successful imprinting.

\subsection{AFM characterization of the surface of the biosensor}

The morphological analysis of the surface of the NIP and MIP after protein removal (MIP rem) modified SPCEs was performed by AFM measurement in liquid medium, because rebinding occurs in this condition (Fig. S4). Even though both samples revealed a rather heterogeneous rough microstructure probably due to the swollen state of the polymeric materials in the liquid environment associated with the inherent roughness of the SPCE substrate, morphological differences between the "hill' and the "valley" regions can also be seen in the images. The NIP surface revealed larger grain profile while the surface of the 'MIP rem' modified SPCE revealed smaller and more numerous

Table 1

Characteristic Raman spectral features of the SPCE analyzed.

\begin{tabular}{llllllllll}
\hline SPCE & $\mathrm{D} / \mathrm{cm}^{-1}$ & $\mathrm{G} / \mathrm{cm}^{-1}$ & $\mathrm{I}_{\mathrm{D}} /$ a.u. & $\mathrm{I}_{\mathrm{G}} /$ a.u. & $\mathrm{I}_{\mathrm{D}} / \mathrm{I}_{\mathrm{G}}$ & $1 / \mathrm{cm}^{-1}$ & $2 / \mathrm{cm}^{-1}$ & $\mathrm{I}_{1} /$ a.u. & $\mathrm{I}_{2} /$ a.u. \\
\hline Pristine & 1352 & 1600 & 109 & 109 & 1,00 & - & - & - & - \\
Blank & 1365 & 1586 & 155 & 165 & 0,94 & 1438 & 1509 & 179 & 135 \\
MIP & 1359 & 1591 & 145 & 156 & 0,93 & 1436 & - & 127 & - \\
MIP & 1370 & 1595 & 131 & 131 & 1,00 & 1437 & 1508 & 137 & 101 \\
$\quad$ rem & & & & & & & & & - \\
NIP & 1351 & 1596 & 166 & 186 & 0,89 & - & - & - & - \\
\hline
\end{tabular}


features/grains. Thus, the average surface roughness (RMS) of NIP and (MIP rem) modified SPCEs were $43 \pm 15 \mathrm{~nm}$ and $37 \pm 11 \mathrm{~nm}$, respectively. These differences were consistent with the role of the template in the imprinting process and the exit of the protein from the polymeric layer, that due to its rather small globular size (unit cell dimensions for IL-1 $\beta$ molecule were estimated to be $5.5 \times 5.5 \times 7.7 \mathrm{~nm}[28]$ ) gave rise to the smaller grain irregular pattern observed at the surface of the modified SPCE. It should be highlighted that these images do not intend to show the empty "cavities" left by the removal of the template due to the swollen state of the polymeric materials, as explained before, and that the information revealed by these are mere suggestions of the observed differences.

\section{5. $\mathrm{pI}$ characterization of IL-1 $\beta$ by LC-MS/MS}

The proteomic analyses applied to a sample of the commercial IL-1 $\beta$ used to assemble the MIP modified SPCE biosensors was made in order to validate the template condition. The LC-MS/MS procedure involved the analysis of several peptide fragments [22] and revealed the presence of just one relevant protein in the tested sample and $97 \%$ aminoacids (153) were identified with high confidence as belonging to human IL$1 \beta$ by proper match with database (accession number B5BUQ8, from UniProt). Moreover, it was possible to determine its MW, as $17.4 \mathrm{kDa}$, and its isoelectric point (pI), of 6.15. These results (MW and aminoacids) are in good agreement with the information provided by the product details sheet (Product No. ABIN2868340 - Interleukin 1, beta (IL1B) (AA 117-269) Protein) sent by the supplier, confirming the high purity of the protein and minimizing a potential contamination risk. The pI value was similar to the ones found in the literature [29] and this was important to be confirmed since the charge distribution of the protein plays a major role not only in the biosensor recognition capability, but also in the polymer imprinted film formation, conditioning the interaction with the redox probe, as seen before.

\subsection{Analytical features of the MIP modified SPCE biosensor}

After the assembly of the MIP modified SPCE, its detection capabilities as biosensor were tested by evaluating its template (IL-1 $\beta$ ) rebinding ability. The characterization of the analytical performance of the biosensor was performed by incubating (for $30 \mathrm{~min}$ ) the modified (MIP or NIP) working electrode SPCE sensing surfaces, with a drop of IL-1 $\beta$ standard solutions of increasing concentrations ( $0.6 \mathrm{pM}$ to $600 \mathrm{nM}$ ) in PBS. After each incubation, the response of the modified SPCE was evaluated in the presence of the ferri/ferrocyanide probe by following the probe electrical features by EIS.

\subsubsection{Linear range and detection limit in buffer}

In order to obtain a calibration curve for the optimized MIP biosensor, the EIS response data $\left(R_{c t}\right)$ was plotted against the logarithm concentration of IL-1 $\beta$, in M (Fig. 3). Error bars were calculated from the standard deviation of the measurements carried out for each concentration. The calibration curve showed a good linear relationship $\left(\mathrm{R}^{2}=\right.$ 0.9991 and $n=5)$ with a slope of $(-443.65 \Omega$ /decade IL-1 $\beta)$ in the range of $60 \mathrm{pM}$ to $600 \mathrm{nM}$ (Nyquist details in Fig. S5). For the more diluted samples tested, the sensor response was not significantly different from the blank. Therefore, based on this calibration curve, the detection
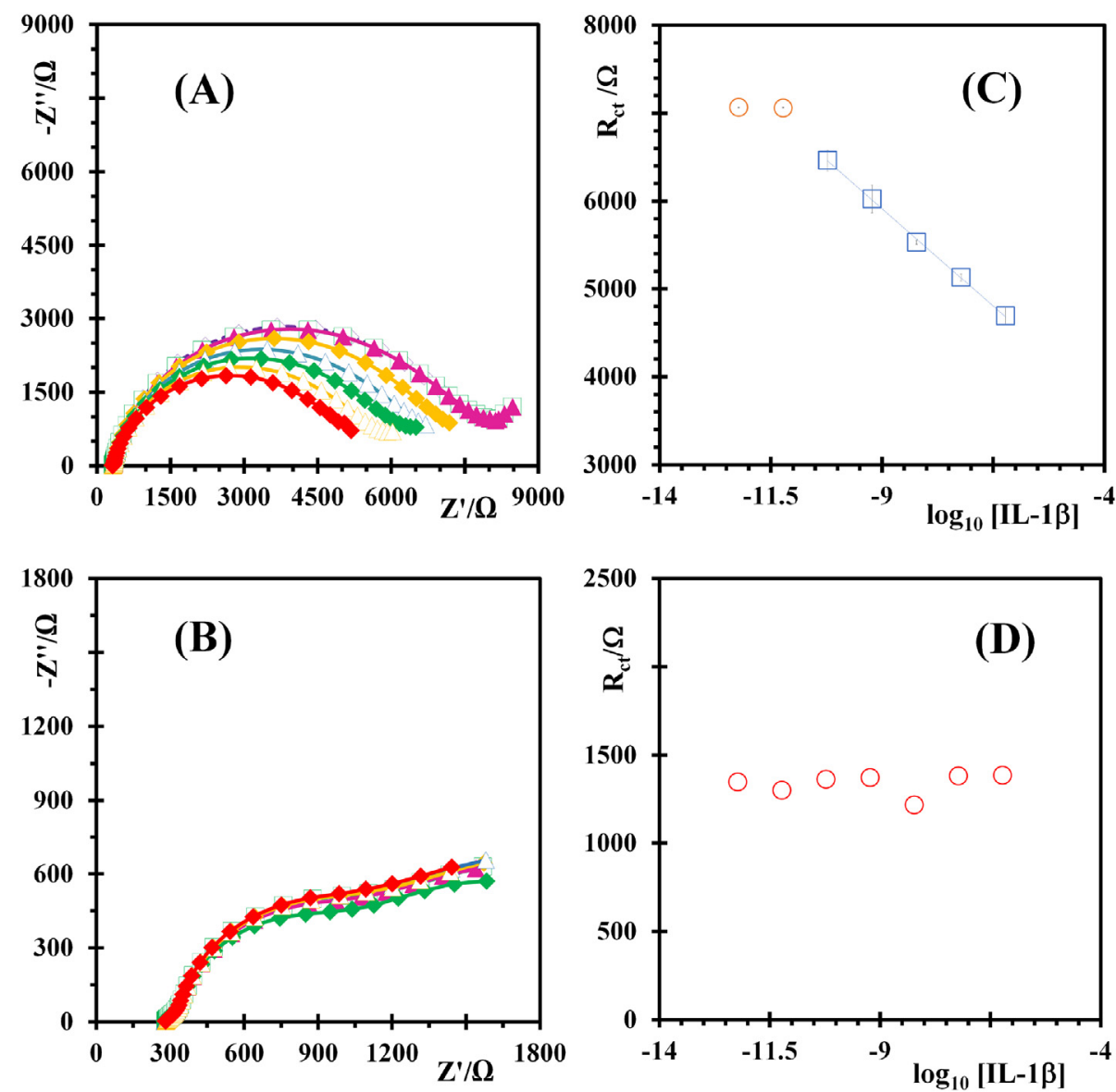

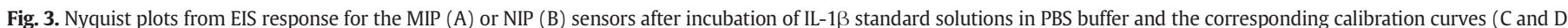

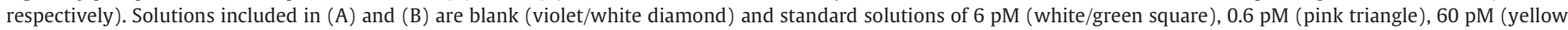

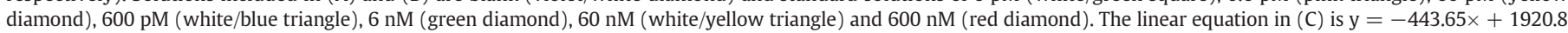
and $\mathrm{R}^{2}=0.9991$. (For interpretation of the references to colour in this figure legend, the reader is referred to the web version of this article.) 

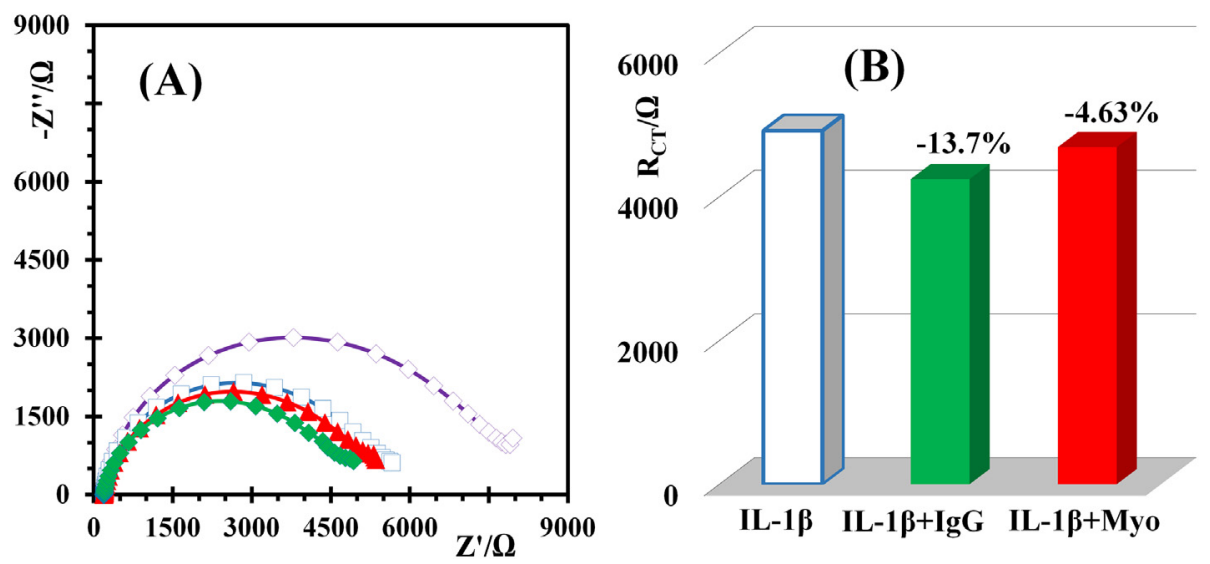

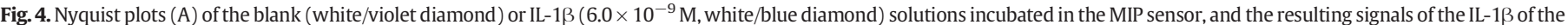

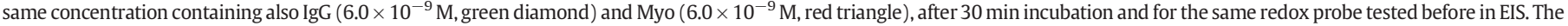
bar graphic (B) shows the corresponding Rct values. (For interpretation of the references to colour in this figure legend, the reader is referred to the web version of this article.)

limit (LOD) was estimated in $1.5 \mathrm{pM}$ with $(\mathrm{S} / \mathrm{N}=3)$. For comparison purposes, the NIP modified SPCE were tested and revealed no correlation with the logarithm of IL-1 $\beta$ concentration.

\subsubsection{Selectivity}

The interference of other proteins were used to test the selectivity of the imprinted sensor by performing the EIS reading of the MIP modified SPCE in the presence of spiked competitive proteins. Myo and IgG were employed for this purpose. Myo was selected due to its similar size and shape to IL-1 $\beta$, with a MW of $17.0 \mathrm{kDa}$ and a pI of 7.36 [30]. IgG was used because it is a relevant competing protein in serum, being the most abundant immunoglobulin, with a MW of $150 \mathrm{kDa}$ and a $\mathrm{pI}$ in the range 7.5-7.8 [31]. These proteins were tested independently, each in binary solutions with IL-1 $\beta$ and compared to that of a single IL- $1 \beta$ solution. The obtained results are shown in Fig. 4 and indicated a higher interference from $\operatorname{IgG}(-13.7 \%)$ than Myo $(-4.6 \%)$ which can be attributed to the large size of the IgG that should be hindering the access of IL-1 $\beta$ to the rebinding cavities of the biosensor. The fact that Myo, a small protein like IL-1 $\beta$ showed lower binding ability indicated good selectivity features of the MIP film.

\subsubsection{SPCE performance in spiked serum}

The characterization of the rebinding ability of the MIP biosensor was also tested in artificial serum conditions in order to check the influence of the medium. Herein, the obtained data was similar to the one obtained in PBS buffer (Fig. 5). Again, the NIP sensor showed no specific
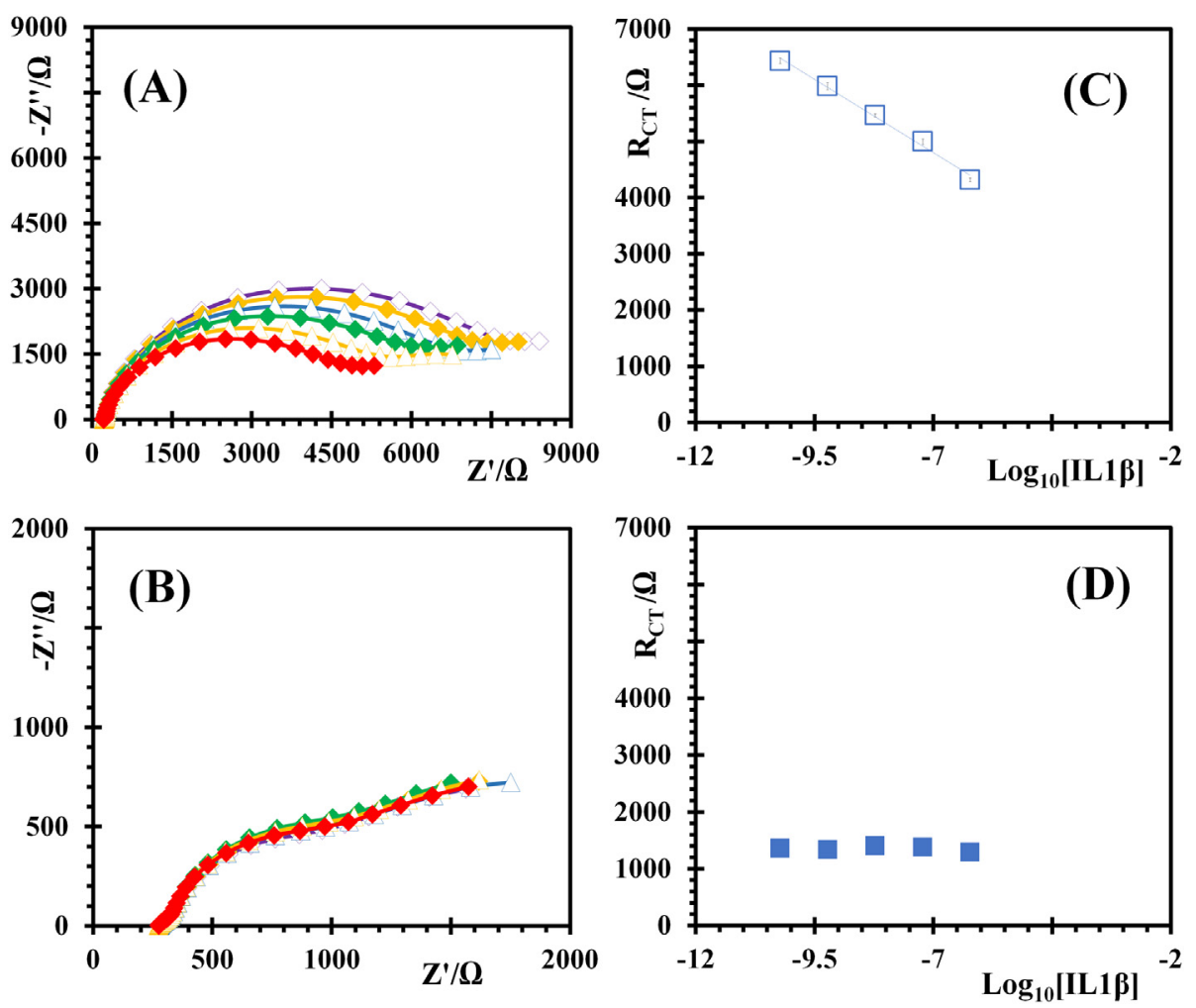

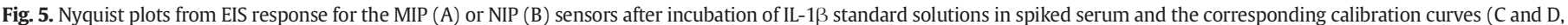

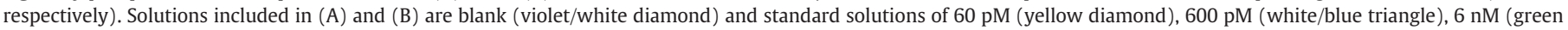

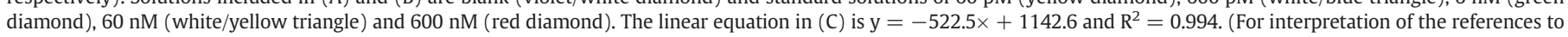
colour in this figure legend, the reader is referred to the web version of this article.) 
response and the MIP response allowed a linear calibration curve $\left(\mathrm{R}^{2}=\right.$ 0.994 and $n=5$ ) with a slope of $-522.5 \Omega$ /decade IL- $1 \beta$ (Nyquist details in Fig. S6). Hence, this allowed the inference that the serum matrix (salts and other components) had only a residual effect in the analytical signal given by the biosensor.

\section{Conclusions}

This work reports for the first time the preparation of a MIP electrochemical sensor for IL-1 $\beta$ detection. Commercial available SPCE electrodes were used to the fabrication of the sensor, because these require small amounts of reagents, are small, cheap and disposable. SPCEs were initially modified by insertion of a pre-polymerized PEDOT modified with 4-AMP layer to increase the peak current signal, enhancing the sensitivity of the MIP assembly. The MIP layer was constructed on top of the modified SPCE by electropolymerization of EBT in the presence of IL-1 $\beta$. The formation of the film was confirmed by Raman and AFM analysis, and EIS was used to show the differences between MIP and NIP sensors. The proposed MIP based device showed a detection limit of $1.5 \mathrm{pM}$ over the range of $60 \mathrm{pM}$ to $600 \mathrm{nM}$ IL-1 $1 \beta$ concentration, tested in PBS. Determination of IL-1 $\beta$ in spiked synthetic serum was also successfully carried out in the same linear concentration range. The device also exhibited high selectivity in discriminating IL-1 $\beta$ from other structurally related compounds (Myo and IgG). Overall, the biosensor showed good analytical results with the advantage of being a simple and quick fully portable platform for on-site screening of IL$1 \beta$, with low sample/reagent consumption when compared to conventional methods.

Furthermore, the use of inexpensive SPCE as a basis for these biosensors will allow simple instrumentation, disposable and portability at low cost. Therefore, this work also demonstrates a new approach to develop a sensitive and label free impedimetric MIP biosensor for IL-1 $\beta$ based on the use of SPE for applications in clinical diagnosis.

\section{Competing interests}

The authors declare that they have no competing interests.

\section{Authors' contributions}

Ana R. Cardoso performed all electrochemical experiments, was involved in data analysis and in draft revision. M. H. de Sá was involved in material characterization, data analysis and made the first draft of the manuscript. M. Goreti F. Sales conceived the study, supervised the experiments and corrected the final draft. All authors read and approved the final version of the manuscript.

\section{Acknowledgements}

This work was funded by the European Union's Horizon 2020 research and innovation program through H2020-FET-Open, under the Project SYMBIOTIC (Grant Agreement: 665046). The authors also acknowledge the support from RESOLVE, a health technology-based program hosted by i3S - Instituto de Investigação e Inovação da Universidade do Porto/Institute for Research and Innovation in Health Sciences of the University of Porto, Portugal, and the access to facilities (namely, the Proteomics Core Facility, where Hugo Osório performed the LC-MS/MS analysis presented here and the Biointerfaces and Nanotechnology Unit, where AFM images of the molecular imprinted materials were taken by Manuela Brás).

\section{Appendix A. Supplementary data}

Supplementary data to this article can be found online at https://doi. org/10.1016/j.bioelechem.2019.04.017.

\section{References}

[1] S. Kuninaka, T. Yano, H. Yokoyama, Y. Fukuyama, Y. Terazaki, T. Uehara, T. Kanematsu, H. Asoh, Y. Ichinose, Direct influences of pro-inflammatory cytokines (IL-1b, TNF-a, IL-6) on the proliferation and cell-surface antigen expression of cancer cells, Cytokine 12 (2000) 8-11, https://doi.org/10.1006/cyto.1998.0504.

[2] Y. Yako, D. Kruger, M. Smith, M. Brand, Cytokines as biomarkers of pancreatic ductal adenocarcinoma: a systematic review, PLoS One 11 (5) (2016), e0154016. https:// doi.org/10.1371/journal.pone.0154016.

[3] S. Chung, D. Geerts, K. Roseman, A. Renaud, L. Connelly, Osteoprotegerin mediates tumor promoting effects of Interleukin-1beta in breast cancer cells, Mol. Cancer 16 (2017) 27, https://doi.org/10.1186/s12943-017-0606-y.

[4] G. Liou, Inflammatory cytokine signalling during development of pancreatic and prostate cancers, J Immunol Res (2017) https://doi.org/10.1155/2017/7979637 Article ID 7979637.

[5] M. Barber, J. Powell, S. Lynch, K. Fearon, J. Ross, A polymorphism of the interleukin$1 \beta$ gene influences survival in pancreatic cancer, Br. J. Cancer 83 (11) (2000) 1443-1447, https://doi.org/10.1054/bjoc.2000.1479.

[6] F. Arduini, L. Micheli, D. Moscone, G. Palleschi, S. Piermarini, F. Ricci, G. Volpe, Electrochemical biosensors based on nanomodified screen-printed electrodes: recent applications in clinical analysis, TrAC Trends Anal. Chem. 79 (2016) 114-126, https://doi.org/10.1016/j.trac.2016.01.032.

[7] M. Trojanowicz, Impact of nanotechnology on design of advanced screen-printed electrodes for different analytical applications, Trends Anal. Chem. 84 (2016) 22-47, https://doi.org/10.1016/j.trac.2016.03.027.

[8] S. Fletcher, Screen-printed carbon electrodes, in: R. Alkire, P. Bartlett, J. Lipkowski (Eds.), Electrochemistry of Carbon Electrodes, Wiley-VCH, Weinheim, Germany 2015, pp. 425-444, (ISBN: 978-3-527-33732-3).

[9] E. Sánchez-Tirado, C. Salvo, A. González-Cortés, P. Yáñez-Sedeño, F. Langa, J. Pingarrón, Electrochemical immunosensor for simultaneous determination of interleukin-1 beta and tumor necrosis factor alpha in serum and saliva using dual screen printed electrodes modified with functionalized double walled carbon nanotubes, Anal. Chim. Acta 959 (2017) 66-73, https://doi.org/10.1016/j.aca.2016. 12.034.

[10] M. Frasco, L. Truta, M. Sales, F. Moreira, Imprinting technology in electrochemical biomimetic sensors, Sensors 17 (3) (2017) 523, https://doi.org/10.3390/s17030523.

[11] R. Elshafey, A. Radi, Electrochemical impedance sensor for herbicide alachlor based on imprinted polymer receptor, J. Electroanal. Chem. 813 (2018) 171-177, https:// doi.org/10.1016/j.jelechem.2018.02.036.

[12] Z. Guoa, A. Florea, C. Cristea, F. Bessueille, F. Vocanson, F. Goutaland, A. Zhang, R. Săndulescu, F. Lagarde, N. Jaffrezic-Renault, 1,3,5-Trinitrotoluene detection by a molecularly imprinted polymersensor based on electropolymerization of amicroporous-metal-organic framework, Sensors Actuators B 207 ( (2015) 960-966, https://doi.org/10.1016/j.snb.2014.06.137.

[13] J. Erdőssy, V. Horváth, A. Yarman, F. Scheller, R. Gyurcsányi, Electrosynthesized molecularly imprinted polymers for protein recognition, Trends Anal. Chem. 79 (2016) 179-190, https://doi.org/10.1016/j.trac.2015.12.018.

[14] E. Akyilmaz, O. Kozgus, H. Türkmen, B. Çetinkaya, A mediated polyphenol oxidase biosensor immobilized by electropolymerization of 1,2-diamino benzene, Bioelectrochemistry 78 (2010) 135-140, https://doi.org/10.1016/j.bioelechem. 2009.09.003.

[15] E.V. Suprun, H.C. Budnikov, G.A. Evtugyn, Kh.Z. Brainina, Bi-enzyme sensor based on thick-film carbon electrode modified with electropolymerized tyramine, Bioelectrochemistry 63 (2004) 281-284, https://doi.org/10.1016/j.bioelechem. 2003.10.025.

[16] A. Tavares, M. Sales, Novel electro-polymerized protein-imprinted materials using Eriochrome black T: application to BSA sensing, Electrochim. Acta 262 (2018) 214-225, https://doi.org/10.1016/j.electacta.2017.12.191.

[17] A. Cardoso, A. Tavares, M. Sales, In-situ generated molecularly imprinted material for chloramphenicolelectrochemical sensing in waters down to the nanomolar level, Sensors Actuators B 256 (2018) 420-428, https://doi.org/10.1016/j.snb.2017. 10.114.

[18] X. Liu, L. Luo, Y. Ding, Z. Kang, D. Ye, Simultaneous determination of L-cysteine and L-tyrosine using Au-nanoparticles/poly-eriochrome black T film modified glassy carbon electrode, Bioelectrochemistry 86 (2012) 38-45, https://doi.org/10.1016/j. bioelechem.2012.01.008.

[19] S. Alvi, M. Hammami, Validated HPLC method for determination of caffeine level in human plasma using synthetic plasma: application to bioavailability studies, J. Chromatogr. Sci. 49 (2011) 292-296, https://doi.org/10.1093/chrsci/ 49.4.292.

[20] A. Bard, L. Faulkner, Electrochemical Methods, Fundamentals and Applications, 2nd ed. John Wiley and Sons, Inc, USA, 2001 (ISBN: 978-0-471-04372-0).

[21] N.A. El-Maali, Voltammetric analysis of drugs, Bioelectrochemistry 64 (2004) 99-107, https://doi.org/10.1016/j.bioelechem.2004.03.003.

[22] J. Daniels, N. Pourmand, Label-free impedance biosensors: opportunities and challenges, Electroanalysis 19 (12) (2007) 1239-1257, https://doi.org/10.1002/elan. 200603855.

[23] I. Horcas, R. Fernández, J. Gómez-Rodríguez, J. Colchero, J. Gómez-Herrero, A. Baro, WSXM: a software for scanning probe microscopy and a tool for nanotechnology, Rev. Sci. Instrum. 78 (2007), 013705. https://doi.org/10.1063/1.2432410.

[24] Q. Zhao, R. Jamal, L. Zhang, M. Wang, T. Abdiryim, The structure and properties of PEDOT synthesized by template-free solution method, Nanoscale Res. Lett. 9 (2014) 557, https://doi.org/10.1186/1556-276X-9-557.

[25] A. Tavares, N. Ferreira, L. Truta, M. Sales, Conductive paper with antibody-like film for electrical readings of biomolecules, Sci. Rep. 6 (2016), 26132. https://doi.org/ 10.1038/srep26132. 
[26] S. Somani, C. Chng, C. Verma, Hydration of a hydrophobic cavity and its functional role: a simulation study of human interleukin-1ß, PROTEINS 67 (2007) 868-885, https://doi.org/10.1002/prot.21320.

[27] T. Jawhari, A. Roid, J. Casado, Raman spectroscopic characterization of some commercially available carbon black materials, Carbon 33 (11) (1995) 1561-1565, https://doi.org/10.1016/0008-6223(95)00117-V.

[28] B. Yu, M. Blaber, A. Gronenborn, G. Clore, D. Caspar, Disordered water within a hydrophobic protein cavity visualized by x-ray crystallography, Proc. Natl. Acad. Sci. U. S. A. 96 (1999) 103-108, https://doi.org/10.1073/pnas.96.1.103.

[29] M. Brattsand, T. Egelrud, Purification and characterization of interleukin $1 \beta$ from human plantar stratum corneum. Evidence of interleukin $1 \beta$ processing in vivo not involving interleukin 13 convertase, Cytokine 10 (7) (1998) 495-592, https:// doi.org/10.1006/cyto.1997.0319.

[30] M. Graf, R. García, H. Wätzig, Protein adsorption in fused-silica and polyacrylamidecoated capillaries, Electrophoresis 26 (2005) 2409-2417, https://doi.org/10.1002/ elps.200410360.

[31] M. De, S. Rana, H. Akpinar, O. Miranda, R. Arvizo, U. Bunz, V. Rotello, Sensing of proteins in human serum using conjugates of nanoparticles and green fluorescent protein, Nat. Chem. 1 (2009) 461-465, https://doi.org/10.1038/nchem.334. 